\title{
Frontières
}

\section{Faire sens grâce à nos opérations conscientes et à nos communications}

\section{La notion de sens chez Niklas Luhmann}

\section{Diane Laflamme}

Volume 15, numéro 2, printemps 2003

URI : https://id.erudit.org/iderudit/1073828ar

DOI : https://doi.org/10.7202/1073828ar

Aller au sommaire du numéro

Éditeur(s)

Université du Québec à Montréal

ISSN

1180-3479 (imprimé)

1916-0976 (numérique)

Découvrir la revue

Citer cet article

Laflamme, D. (2003). Faire sens grâce à nos opérations conscientes et à nos communications : la notion de sens chez Niklas Luhmann. Frontières, 15(2), 78-84. https://doi.org/10.7202/1073828ar

\section{Résumé de l'article}

La notion de sens joue un rôle d'importance majeure dans la théorie des systèmes sociaux développée par Niklas Luhmann. Cette courte présentation aborde quelques aspects du sens : la distinction entre les systèmes psychiques et les systèmes sociaux, tous deux étant considérés comme constitutifs du sens, les trois dimensions du sens et le processus de généralisation du sens dans des attentes et des structures. La phénoménologie et l’herméneutique sont interrogées en parallèle pour éclairer le vocabulaire que choisit Luhmann pour aborder la notion de sens et pour décrire une forme originale de constitution du sens dans la mouvance de ces théories. 


\section{Résumé}

La notion de sens joue un rôle d'importance majeure dans la théorie des systèmes sociaux développée par Niklas Luhmann. Cette courte présentation aborde quelques aspects du sens: la distinction entre les systèmes psychiques et les systèmes sociaux, tous deux étant considérés comme constitutifs du sens, les trois dimensions du sens et le processus de généralisation du sens dans des attentes et des structures. La phénoménologie et I'herméneutique sont interrogées en parallèle pour éclairer le vocabulaire que choisit Luhmann pour aborder la notion de sens et pour décrire une forme originale de constitution du sens dans la mouvance de ces théories.

Mots clés : sens - Luhmann - autopoïèse.

\section{Abstract}

The concept of meaning plays a major role in Niklas Luhmann's theory of social systems. Various aspects of meaning are briefly discussed here: the distinction between psychic systems and social systems, both defined as meaning-constituting systems, the three dimensions of meaning and, finally, the process of generalisation of meaning leading to the emergence of expectations and structures. Elements from phenomenology and hermeneutics are also quoted in an attempt to shed more light on Luhmann's vocabulary and to examine how Luhmann used some of the insights gained through these theoretical perspectives to describe in his own way the operations of meaningconstituting systems.

Key words: meaning - Luhmann autopoiesis.

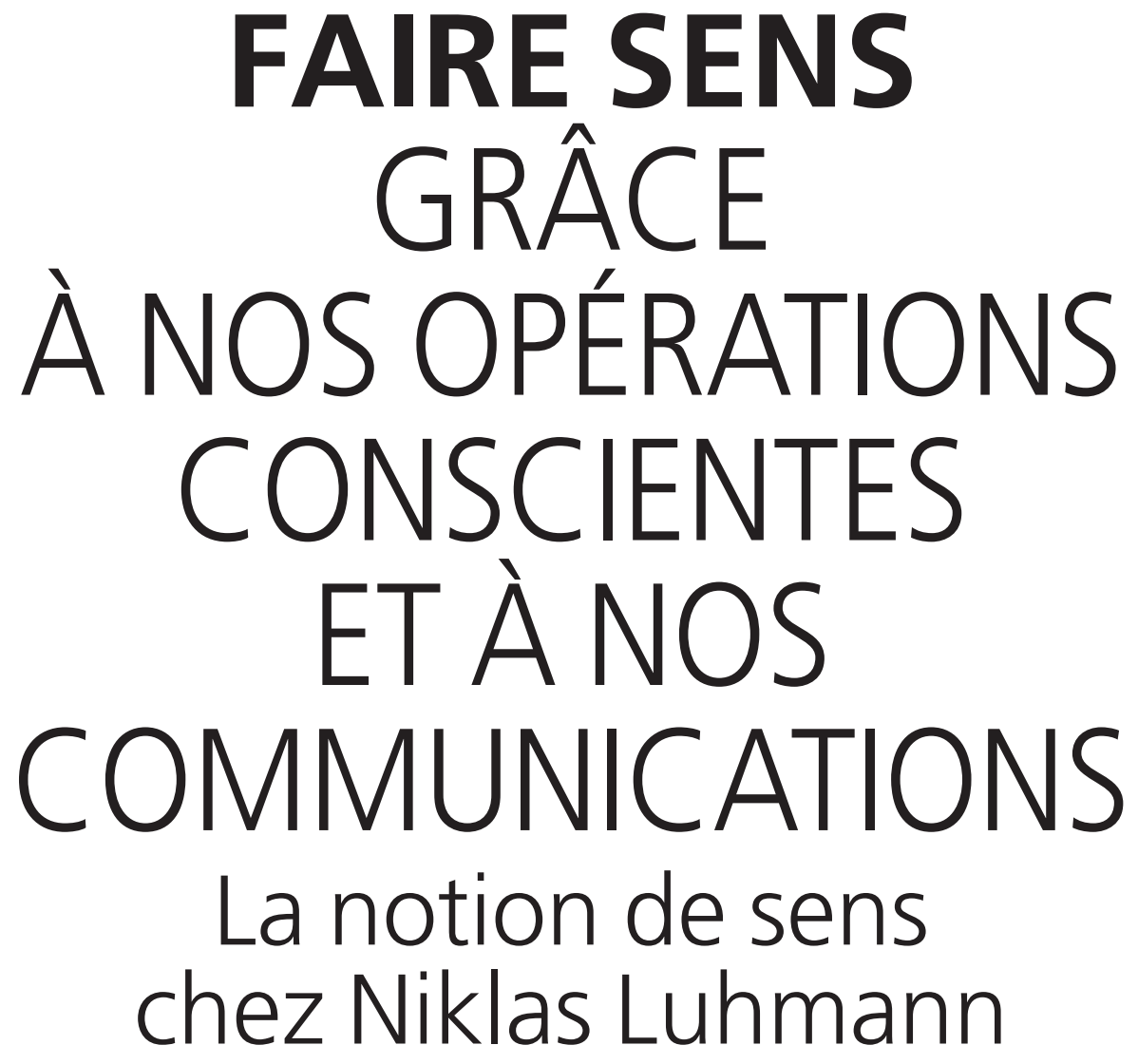

Diane Laflamme, Ph.D., chargée de cours, UQAM.

Le sens, de quoi s'agit-il? Les êtres humains n'en finissent pas de chercher ou d'attribuer un sens à leur existence. Chacun d'entre nous s'interroge périodiquement sur le sens de telle ou telle expérience, de telle ou telle action. Nous attribuons un sens aux mots et aux signes avec lesquels nous essayons de communiquer avec les autres êtres humains. Nous nous interrogeons sur le sens de la mort. Derrière notre notion de sens se profile un vaste paysage, un univers, un monde... " notre » monde, à la fois assez flou pour ne pas trahir nos attentes et assez précis pour que nous tentions inlassablement d'y ouvrir des chemins pour mieux nous orienter... à même nos expériences, nos actions, nos mots, nos signes.
C'est ainsi que le trajet que suit l'émergence du sens serait circulaire ${ }^{1}$. Et c'est en tenant compte de cette circularité que le sociologue allemand Niklas Luhmann (1927-1998) attribue les opérations constitutives de sens à deux catégories de systèmes: les systèmes psychiques et les systèmes sociaux. Ces systèmes ont en commun deux caractéristiques: ils sont l'un et l'autre autoréférentiels, c'est-à-dire qu'ils sont en mesure de se prendre euxmêmes comme référence, comme s'ils étaient distincts d'eux-mêmes, et autopoiétiques, c'est-à-dire qu'ils s'autoproduisent à même leurs propres opérations et uniquement à partir de leurs propres opérations. Les opérations des systèmes psychiques sont des opérations conscientes: les systèmes psychiques produisent des pensées à partir d'autres pensées; les opérations des systèmes sociaux sont des 
communications: les systèmes sociaux produisent de la communication à partir de la communication.

En suivant la piste du sens (meaning, en anglais ; Sinn, en allemand), Luhmann observe des systèmes qui, dit-il, sont euxmêmes des systèmes observateurs, c'est-àdire des systèmes capables d'observer leurs expériences et leurs actions et de les distinguer de ce qui se passe dans ce qui est du même coup désigné par le système observateur comme son environnement. Nous commencerons par situer la classification des systèmes que propose Luhmann en nous demandant où le sens est constitué et nous poursuivrons ensuite notre exploration de la notion de sens selon Luhmann, en décrivant les opérations qui permettent la constitution du sens en situation de complexité et en présentant brièvement les trois dimensions du sens ainsi que le mécanisme de généralisation du sens dans des attentes et des structures.

Luhmann est généralement considéré comme un théoricien d'accès difficile. En effet, il nous oblige à remettre en question la plupart des outils d'abstraction dont nous avons hérité (entre autres de la tradition philosophique occidentale) pour construire «notre» monde. Je dis bien construire, car cette théorie se situe dans la mouvance du constructivisme: Luhmann la présente comme un constructivisme axé sur les opérations (operational constructivism, 2000, p. 6 et 91). Il n'en demeure pas moins que certains des concepts que l'on retrouve chez Luhmann avaient déjà inspiré ailleurs des tentatives d'élucidation, notamment dans les études sur le sens que proposent la phénoménologie d'inspiration husserlienne et l'herméneutique inaugurée par Dilthey. Luhmann y fait d'ailleurs ouvertement référence et nous puiserons dans ces deux traditions philosophiques afin d'indiquer d'où provient le vocabulaire dont Luhmann se sert pour aborder la notion de sens et pour élaborer, surtout en rupture et parfois en continuité, sa propre théorie de la constitution du sens.

\section{OÙ LE SENS EST-IL CONSTITUÉ ?}

Pour les êtres humains, la vie, les choses ont un sens. N'est-ce pas là ce qui nous distingue des machines intelligentes que nous sommes maintenant en mesure de construire? Les premiers théoriciens $\mathrm{du} \mathrm{XX}^{\mathrm{e}}$ siècle qui se sont réclamés de la cybernétique puis de la Théorie des systèmes s'intéressaient d'ailleurs à des machines. Pour élaborer sa présentation des systèmes psychiques et des systèmes sociaux, Luhmann situe sa démarche à l'intérieur d'un nouveau paradigme (1995, Paradigm Change in Systems Theory, p. 1-11), inauguré par les travaux de von
Bertalanffy. Un système ne peut plus être envisagé comme un contenant rempli d'objets ou de relations. Pour Luhmann un système n'est pas un objet mais une distinction ; un système ne se définit pas comme un tout comportant des parties mais bien plutôt comme une unité qui ne peut être distinguée sans faire référence à un environnement.

La théorie des systèmes de Luhmann est axée sur des opérations de différenciation La distinction entre système et environnement est le principal outil conceptuel proposé par Luhmann, un outil qui selon lui aurait une portée aussi prometteuse que les catégories d'Aristote (1990b, p. 282)! Donc le système est une distinction; il peut observer d'autres distinctions qui sont de type système / environnement comme lui et... il peut s'observer lui-même en tant que distinction système / environnement. C'est là le champ d'étude de la cybernétique de deuxième génération, l'observation des systèmes observateurs.

Un système se caractérise par ses opérations; pour acquérir des éléments de connaissance au sujet d'un système, il nous faudra donc observer ses opérations. Or en observant les opérations des systèmes psychiques et des systèmes sociaux, Luhmann constate qu'elles sont « constitutives de sens» (meaning-constituting operations), alors que le sens ne joue aucun rôle dans les opérations des machines et des organismes.

Luhmann observe aussi qu'il y a interpénétration entre les systèmes biologiques vivants, les systèmes psychiques conscients et les systèmes sociaux. Les systèmes biologiques constituent un environnement indispensable pour les opérations des systèmes psychiques conscients et les systèmes psychiques constituent un environnement indispensable aux opérations de commu- nication des systèmes sociaux. S'il n'y a pas d'êtres humains vivants, il n'y aura ni systèmes psychiques conscients ni systèmes sociaux offerts à notre observation. Ces trois catégories de systèmes ont une organisation autopoiétique. Le tableau 1, présente l'autopoïèse ${ }^{2}$ axée sur la production de la vie et l'autopoïèse axée sur la production du sens. Parmi les systèmes sociaux, Luhmann distingue aussi entre les interactions, les organisations et la société.

L'interpénétration entre les trois catégories d'organisation autopoiétique produit des relations de dépendance entre les systèmes en cause ; ces relations de dépendance réussissent à persister dans le temps pourvu qu'elles contribuent à l'évolution des systèmes placés en interpénétration. Luhmann ajoute que c'est le sens qui permet qu'il y ait interpénétration entre les systèmes psychiques et les systèmes sociaux. Le sens permet à la conscience de se comprendre elle-même tout en continuant de s'autoproduire en communiquant et, en même temps, le sens permet que la communication soit référée à la conscience des participants (1995, p. 219).

Où sont les êtres humains dans tout cela ? Luhmann l'écrit clairement : un être humain n'est pas un système (1995, p. 40 et 255-277). Plus encore, il est impossible, selon Luhmann, d'obtenir un système en rassemblant à l'intérieur d'un tout plusieurs êtres humains. La société n'est pas un contenant rempli d'individus! Les éléments constitutifs d'un système social sont des communications et non pas des personnes.

Avec le terme système psychique, Luhmann ne prétend pas rendre compte de toute la complexité vivante qu'est un être humain ; il indique plutôt que certaines opérations humaines peuvent être observées et attribuées à un système qui produit des pensées conscientes à partir d'autres

\section{Tableau 1 - DEUX MODES D'ACTUALISATION DE L'ORGANISATION AUTOPOÏÉTIQUE}

\begin{tabular}{|c|c|c|}
\hline $\begin{array}{l}\text { Autopoïèse : } \\
\text { Produire de la vie } \\
\text { à partir de la vie }\end{array}$ & \multicolumn{2}{|c|}{$\begin{array}{l}\text { Autopoïèse : } \\
\text { Produire du sens } \\
\text { à partir du sens }\end{array}$} \\
\hline $\begin{array}{l}\text { Les systèmes vivants } \\
\text { (cellules, organismes) }\end{array}$ & $\begin{array}{l}\text { Les systèmes } \\
\text { psychiques conscients } \\
\text { (dont les opérations } \\
\text { sont des pensées propres } \\
\text { qui produisent d'autres } \\
\text { pensées propres) }\end{array}$ & $\begin{array}{l}\text { Les systèmes sociaux } \\
\text { (dont les opérations sont des } \\
\text { communications qui produisent } \\
\text { d'autres communications) : } \\
\text { - les interactions (un système } \\
\text { social qui émerge entre ceux } \\
\text { qui sont présents l'un à l'autre); } \\
\text { - les organisations (des systèmes } \\
\text { sociaux qui peuvent communi- } \\
\text { quer en tant qu'appareils); } \\
\text { - la société (la totalité de toutes } \\
\text { les communications sociales } \\
\text { auxquelles on peut s'attendre). }\end{array}$ \\
\hline
\end{tabular}


pensées conscientes, et qui s'attribue à luimême ces pensées conscientes. Luhmann propose de parler d'une «personne» pour désigner un système psychique en tant que ce système est alors observé par un autre système psychique ou par un système social (1995, p. 109). L'observation de cette complexité qu'est l'être humain se fait par le biais d'observations toujours partielles, qui ne prétendent pas épuiser cette unité vivante.

Avec le terme communication, Luhmann fait référence à une synthèse complexe, qui ne se démarque pas vraiment de ce que recouvre habituellement ce mot dans son usage courant mais qui comporte précisément l'avantage de nous obliger à observer toute la complexité de ce que nous tenons pour acquis lorsque nous disons «avoir communiqué ». Pour qu'il y ait communication, il nous faut trois choses : un élément d'information est donné par une opération de distinction, quelque chose est formulé et quelque chose est compris. La communication n'est pas un fluide hypothétique qui circulerait entre un émetteur et un récepteur; elle est une opération de synthèse, elle est l'événement qui se produit lorsque le système en cause réussit à connecter en une synthèse l'information, la formulation et la compréhension. C'est parce que la communication est constamment obligée de sélectionner une autre communication pour poursuivre la communication que le système en cause poursuit son autopoïèse (son autoproduction), qu'il continue d'exister en tant que système social, c'est-à-dire qu'il continue de constituer du sens en produisant de la communication à partir d'autres communications. Les éléments constitutifs d'un système social, ce ne sont pas les personnes en cause, ce sont ces événements, ces synthèses appelées communications.

Aussitôt qu'un système social ne réussit plus à connecter sa communication à d'autres communications, la communication cesse et ce système social cesse d'exister, de s'autoproduire. Aussitôt qu'un système psychique ne réussit plus à connecter ses pensées propres à d'autres pensées propres dans un processus conscient, la conscience cesse de s'autoproduire.

\section{AUTOPOÏËSE ET MORT}

Notre système psychique peut être éveillé, ou dormir... peut-il mourir ? Luhmann écrit à ce sujet qu'un système psychique conscient ne peut envisager la cessation de son autopoiièse, c'est-à-dire la cessation de la production de la conscience à partir de la conscience. Citant à cet égard Jean-Paul Sartre, ainsi que cette belle phrase de Paul Valéry: La mort est une surprise que fait l'inconcevable au concevable ${ }^{3}$, Luhmann écrit que nous pouvons imaginer notre propre mort comme étant la fin de la vie, mais pas comme la fin de la conscience.

Tous les éléments de la conscience (c.-à-d. toutes les opérations d'un système conscient ; ces élément ou opérations sont des événements évanescents) sont axés sur la reproduction de la conscience maintenant et «ainsi de suite». Nier cet « ainsi de suite » reviendrait pour la conscience à produire un élément, un événement qu'elle ne réussirait pas à reconnaître au nombre des éléments connectés par le système psychique, précisément parce que la raison d'être du réseau de connexions que produit la conscience est de permettre l'autoproduction de la conscience.

Il n'y pas de place dans ce réseau de connexions pour un événement conscient qui aurait la caractéristique d'être « le dernier de la série ». Le système psychique envisagera plutôt l'éventualité d'une interruption de son autopoïèse comme un "épisode » et évoquera la possibilité de «sauter» (leap) d'un épisode à l'autre. Qu'il s'agisse de l'autopoïèse axée sur la vie ou de celle axée sur le sens, un processus autopoiétique n'est pas un processus orienté vers une « opération finale » ou un « point final » que le système pourrait envisager comme tel ; l'autopoïèse du système en cause se continue ou s'arrête, c'est tout (1995, p. 276 ; voir aussi 1990a, p.118). D'où l'intérêt de savoir que l'être humain n'est pas un système; l'observer en tant que système nous renseignera sur des aspects fascinants, entre autres, sur la constitution du sens grâce à la conscience et à la communication humaines, mais ne nous donnera pas pour autant accès à la condition humaine dans toute sa complexité.

\section{AUTOPOÏËSE}

\section{ET PHÉNOMÉNOLOGIE}

Certes, la phénoménologie ne parle pas d'autopoïèse ni de système psychique qui s'autoproduit en produisant du sens; elle parle plutôt de genèse transcendantale et d'autoconstitution du je, du sujet qui tout en constituant le monde se constitue progressivement lui-même et s'apparaît à luimême, devient conscient de lui-même. Husserl écrit dans la $4^{\mathrm{e}}$ Méditation cartésienne: "Mais il faut remarquer que le je qui fonctionne comme centre n'est pas un pôle d'identité vide (pas plus que ne l'est un objet quelconque), mais en vertu d'une légitimité propre à la genèse transcendantale, il acquiert avec chacun de ses actes qui émanent de lui un nouveau sens objectif, une nouvelle propriété permanente. Si par exemple, je me décide pour la première fois, dans un acte de jugement, pour un être et un être tel, cet acte fugitif passera, mais désormais, je suis et je reste ce moi qui s'est décidé de telle ou telle manière [...]» (1994, [32] p. 113). Luhmann ne retiendrait pas ici le caractère transcendantal $^{4}$ de la «genèse» du je que décrit Husserl, mais on constate que les systèmes autopoiétiques qu'il observe ont eux aussi comme éléments des événements « fugitifs » et il observerait un système qui « instruit une distinction » là où Husserl décrit un je qui pose un «acte de jugement »; il observerait un système qui opte pour telle ou telle "sélection », au lieu de dire comme Husserl que le je "s'est décidé de telle ou telle manière ».

Les éléments du système sont des opérations ; ces opérations sont elles-mêmes des événements et non pas des objets, elles n'ont aucune permanence : l'opération est un événement évanescent. Cette façon de voir les choses transforme en profondeur la notion d'être ; là où la philosophie occidentale a tant cherché une substance, on se retrouve plutôt avec des «dévolutions répétées allant d'un moment à l'autre ». Jean Clam, dans un commentaire sur cet aspect de la théorie de Luhmann, l'exprime en ces termes : «Or, en y regardant de près, l'on se rend compte que cette évanescence, au lieu de représenter une diminution ontologique de l'actualité des systèmes, est bien au contraire un moyen de promouvoir la permanence systémique, par le biais du report qu'elle occasionne des garanties externes de celle-ci vers une architecture extrêmement fine de soutènement du système dans "l'être". En effet, on passe, avec elle, d'assomptions lourdes postulant une permanence substantielle, unitaire et massive, vers des dévolutions répétées allant d'un moment à l'autre, sauvant le système d'un événement de soi à l'autre, et permettant l'avènement de condensations et de décondensations appropriées aux synthèses courantes du système » (1997, p. 257).

\section{LA CONSTITUTION DU SENS EN SITUATION DE COMPLEXITÉ}

Luhmann désigne à notre attention des systèmes dont les opérations permettent la constitution du sens (meaning-constituting systems, 1990a, p. 23). Il ajoute qu'il y a constitution du sens dans et par l'action et l'expérience (meaning-constituting experience and action) humaines. Il y a action lorsque le système s'attribue à lui-même l'opération observée, alors qu'il s'agit d'une expérience si l'opération est attribuée à l'environnement.

Le système opère en situation de complexité, la notion de complexité servant ici à indiquer qu'il existe toujours plus de possibilités d'expérience et d'action que ce que le système est en mesure d'actualiser (1990a, p. 26). Le système procède constamment à des choix ; ses opérations sont 
des sélections : ceci plutôt que cela. Ce qui n'est pas sélectionné ne cesse pas pour autant d'exister. Le bassin de possibilités de choix ainsi constitué, ce sera le monde, un monde qui est source inépuisable de nouvelles sélections, un monde dont le système fait l'expérience comme étant multi-dimensionnel (3 dimensions décrites ci-après) alors même que c'est lui qui est en train de le constituer par ses propres opérations.

Le monde est constitué ; le sens est constitué ; le système se constitue. On ne trouve pas chez Luhmann de théorie sur l'existence ou l'essence des systèmes, mais une question : comment le monde et le sens sont-ils constitués par les systèmes qui observent qu'il existe un monde, qu'il existe du sens? Comment un système-observateur du monde, du sens et de sa propre émergence en tant que système en arrive-t-il à se constituer lui-même?

\section{CONSTITUTION}

Pour Luhmann, c'est grâce aux opérations des systèmes psychiques et des systèmes sociaux qu'il $\mathrm{y}$ a « constitution du sens ». On ne peut s'empêcher d'envisager ici un lien avec la phénoménologie husserlienne, où la notion de constitution joue un rôle majeur et où elle désigne l'activité du sujet par laquelle un objet ou un vécu est donné audit sujet et acquiert un sens pour lui ${ }^{5}$. Lorsqu'il se propose à son tour d'utiliser le terme, Luhmann y reconnaît une "catégorie centrale» de la phénoménologie, tout en déplorant le caractère ambivalent que lui aurait donné Husserl (1990a, p. 25 et 69). Comme Luhmann ne retient pas, nous l'avons déjà dit, la dimension transcendantale de la théorie de Husserl, qu'il remplace le couple sujetobjet par la distinction système / environnement et qu'il considère le concept de sens comme étant premier et devant être défini sans faire référence au sujet, sa description des opérations grâce auxquelles le système se constitue, constitue son monde et fait sens s'écarte à maints égards de la notion de constitution chez Husserl (1990a, p. 2223 ; 1995, p. $145-147$ et 512). Il demeure néanmoins intéressant de rappeler brièvement ici comment Husserl présentait le concept de "constitution », dans son sens large:

Au sens plus large du mot, un objet se «constitue»qu'il soit réel ou non »- au sein de certains enchaînements immanents à la conscience qui comportent une unité évidente, dans la mesure où ils entraînent par essence la conscience d'un $\mathrm{X}$ identique

(1950, p. 457).
Le vocabulaire de Husserl dans l'extrait cité, avec des mots comme immanence et essence, est bien loin de celui de Luhmann. Husserl observe pourtant quelque chose de très semblable à ce que Luhmann observe à même les opérations qui permettent la constitution du sens par un système psychique : des enchaînements qui comportent une unité et qui entraînent la reconnaissance d'un identique.

Là où Husserl voyait des enchaînements, Luhmann décrira des distinctions et un incessant processus de sélection : une distinction est une opération qui oblige le système à indiquer ce qu'il retient dans son observation, reléguant du même coup tout le reste (ce qui n'est pas distingué) à l'arrière-plan. Un système dont les opérations sont constitutives de sens est capable de distinguer entre l'actualité de ce qui est distingué et la potentialité de tout ce qui reste, qui n'est pas distingué, qui sort de scène mais qui demeure indissociable de ce qu'indique la distinction, ne serait-ce que parce qu'il s'en démarque. Luhmann constate que chaque fois que nous faisons l'expérience du sens, nous faisons l'expérience d'un donné qui déborde ce qu'il donne: un donné qui se distingue de tout le reste qu'il a exclu tout en le gardant virtuellement accessible à une autre opération de distinction.

\section{INSTITUTION}

La phénoménologie husserlienne décrit un autre mécanisme qu'il est éclairant de rappeler pour le comparer à la proposition de Luhmann. Il s'agit de l'institution du sens. En allemand, Husserl a recours à une série de trois concepts: Stiftung (institution), Urstiftung (institution originaire) et Nachstiftung (réinstitution ou institution après coup), selon les traductions qu'en propose Marc Richir. Ce dernier décrit comme suit le processus en trois temps par lequel il y a institution d'un sens: "Pour Husserl, cette Stiftung d'un sens, englobant toujours des cas réels et possibles, donc des pluralités qui ne sont jamais complètement actualisées renvoie tout à la fois à la première fois, inaugurale, de la Stiftung dans l'Urstiftung, et à la possibilité de la reprise de l'Urstiftung dans la Nachstiftung. [...] L'essentiel à comprendre ici est que, dans toute Stiftung de sens, aussitôt que le sens dans sa vivacité (le sens se faisant comme nous le disons à la suite de Merleau-Ponty) s'est perdu dans l'horizon vide des rétentions vides (vides de toute intuitivité), le sens y est sédimenté, en sommeil, à l'état potentiel, et que, corrélativement, nous avons acquis un habitus correspondant à le réactiver, une disposition à le retrouver et à le faire revivre dans sa vivacité »( Richir, 2001, p.186).
Dans son observation des systèmes dont les opérations sont constitutives de sens, Luhmann retient pour sa part un processus en quatre temps : il définit le sens comme l'unité qui émerge d'un processus ${ }^{6}$ d'actualisation, désactualisation, réactualisation et revirtualisation qui s'alimente luimême, tout en demeurant conditionné par le système porteur du processus (1995, p. 65). On l'a déjà dit, Luhmann aborde le sens comme une "distinction », une différence, plus précisément la différence entre ce qui est actuel à un moment précis et l'horizon de possibilités à partir duquel le système indique ce qu'il distingue. C'est en retournant à cet horizon de possibilités que le système procédera à la distinction suivante, à la sélection suivante de façon à continuer de faire sens dans ses pensées conscientes (système psychique) ou dans sa communication (système social).

Observant ce que nous, êtres humains, appelons notre expérience, Luhmann constate qu'elle fait toujours référence à quelque chose d'autre, en plus de s'actualiser comme «cette» opération consciente ou " cette » opération de communication, plutôt qu'une autre. Ce qu'il appelle « le sens », c'est la solution que propose le système psychique ou le système social alors en cause pour résoudre le problème que cause la coexistence de ces deux aspects dans l'expérience humaine : son contenu actuel et tout ce à quoi l'expérience fait référence en plus de son contenu actuel (1990a, p. 25).

\section{ENVELOPPEMENT}

Luhmann constate qu'en tant que "phénomène ", le sens se constitue comme en excès par rapport à lui-même : "Quelque chose se tient là, au point focal, au centre de l'intention, et tout le reste est indiqué de façon marginale, comme l'horizon d'un « et ainsi de suite» indissociable de l'expérience et de l'action» (1995, p. 60). Il y a un «surplus » de références à d'autres possibilités d'expérience et d'action. Le sens fait référence à : ce qui est indiqué par le système dans le réel (ou ce qui est présumé réel), aux autres possibilités parmi lesquelles le système a fait une sélection (un réel sous conditions) et à ce qui est nié par la sélection (parce que considéré non réel ou impossible). Toutes ces références qui accompagnent un objet de sens font en sorte que le système dont les opérations visent cet objet n'arrive jamais à les actualiser toutes et est ainsi poussé à procéder constamment à de nouvelles sélections parmi ces références.

Avec un enthousiasme qui n'est pas incompatible avec celui de Luhmann pour le «surplus » de références rattaché au sens, Jean-Michel Salanskis (1997, p. 417-418), mentionne l'enveloppement du sens au nombre des caractéristiques de «l'effet-de-sens » 
que le développement de la pensée herméneutique «et plus généralement le mouvement de la pensée moderne» ont permis de dégager : "L'enveloppement du sens est sa structure décisive. ce qui fait que le sens porte l'existence, la temporalise, qu'il passe et qu'il oriente, c'est qu'il est enveloppé en lui-même, que chaque occurrence de l'adresse porte plus de sens qu'elle n'en signifie. Même si aucune élucidation de l'excès du sens dans son occurrence n'a lieu, cet excès est constitutif du sens comme tel. Une occurrence de sens absolument transparente ne serait pas occurrence de sens, mais exhibition d'objet, présentation pleine. »

\section{LES TROIS DIMENSIONS DU SENS}

Notre expérience du monde est multidimensionnelle. Pour faire sens, une opération du système opère une sélection entre des possibilités qui se répartissent selon les trois dimensions de notre expérience du monde, auxquelles correspondent, dit Luhmann, trois dimensions du sens. Plusieurs possibilités de sélection s'offrent au système à l'intérieur de son horizon de sens et ces possibilités ne disparaissent pas du seul fait qu'elles n'ont pas été thématisées par le système (sélectionnées); le système pourra y revenir sans quitter son horizon de sens. Le sens se constitue à partir d'événements évanescents que le système demeure en mesure de connecter entre eux pour poursuivre les seules opérations qui soient à sa portée: des sélections. Ces sélections se répartissent selon une matrice à trois dimensions, ce qui augmente les chances du système de réussir une connexion et de poursuivre ses opérations conscientes ou sa communication (tableau 3).

Chacune des opérations du système cherche à se connecter à une autre opération pour poursuivre le processus autopoïétique. Chaque opération se détermine elle-même selon les possibilités de connexion qui s'offrent à elle dans les trois dimensions du sens (1995, p. 83). Le système fera parfois l'expérience de discontinuités entre les trois dimensions, mais le processus de généralisation symbolique favorisera les attentes qui réussissent à faire le pont en tenant compte de ces discontinuités (1995, p. 97). Le processus devient ainsi porteur d'évolution.

\section{LA GÉNÉRALISATION DU SENS \\ DANS DES ATTENTES}

Ce que Luhmann appelle ici une attente est l'une des «formes » que peut prendre le sens, et non pas un processus psychique interne (1995, p. 293). Il y a généralisation $\mathrm{du}$ sens sous forme d'attentes, d'expectations. La généralisation condense ce à quoi réfère le sens dans des attentes qui permettent au système, pour chaque situation de sens, de prévoir la prochaine sélection qui s'impose. Il y a aussi généralisation d'une attente du système aussitôt que cette attente acquiert une certaine indépendance par rapport à l'événement précis auquel elle se réfère (1995, p. 326). Ce processus de généralisation du sens fait partie d'une chaîne en trois temps : spécification, généralisation, re-spécification (1995, p. 329).

Le processus de généralisation introduit par ailleurs un certain degré d'indétermination : le système ne sait plus aussi clairement à quoi s'attendre, mais l'insécurité sera plus facilement absorbée en se répartissant selon les trois dimensions du sens. Aussitôt que le système distingue qu'une attente n'est plus valide, c'est-à-dire qu'elle ne permet plus à ses opérations de se connecter l'une à l'autre en orientant la sélection qui doit nécessairement suivre chaque sélection opérée par le système, cette attente est abandonnée. Le processus est circulaire : en mettant à l'épreuve ses attentes, le système choisit par ailleurs jusqu'où pousser la généralisation du sens.

Le sens est constitué lors de chaque opération du système grâce à une sélection bien précise, qui a le caractère d'un événement évanescent que le système ne pourra plus répéter comme tel par la suite mais, comme le sens fait aussi référence à des condensations, des sédimentations par thèmes le système pourra s'y référer lors d'autres sélections. Sous l'effet de la généralisation symbolique, des identités viennent se condenser là où il n'y aurait autrement qu'un flux indifférencié d'événements qui perdent constamment leur actualité. C'est un pro- cessus qui s'avère fiable et sur lequel nous comptons sans même nous en rendre compte $^{8}$ (1995, p. 94).

\section{DES CONDENSATIONS STRUCTURELLES}

Les structures du système se forment à partir des attentes (1995, p. 294) ; il y a émergence de condensations structurelles. Luhmann s'intéresse à des systèmes dont les éléments sont, nous l'avons déjà dit, des événements liés l'un à l'autre dans le temps : "Le système consiste seulement en événements temporellement liés, et non pas en composantes fermes (atomes, individus...) » (Luhmann cité dans Clam, 1997, p. 258). Ces éléments du système, ce sont ses opérations. Les opérations du système sont récursives, c'est-à-dire que chaque sélection du système est effectuée à partir de la sélection précédente. Luhmann introduit alors le concept de structure pour indiquer que le système lui-même se donne des contraintes qui orientent ses sélections (1995, p. 282 et 286). Certaines contraintes deviennent des structures pour le système parce qu'elles lui permettent de connecter plus rapidement ou avec moins d'incertitude une sélection à une autre, parmi toutes celles qu'il lui serait possible d'effectuer. Le jeu des attentes produit un tel effet de structure (1995, structures of expectation p. 289 et 294 ; expectational structures, p. 292 ) car, en réactivant une attente qui avait été préalablement obtenue par la condensation d'opérations évanescentes, le système réduit son incertitude quant à la sélection qui doit suivre la sélection qu'il vient d'effectuer, ce qui augmente ses chances de produire une connexion qui continuera de faire sens.

Tableau 2 - LES TROIS DIMENSIONS DU SENS

\begin{tabular}{|c|c|c|c|c|c|}
\hline $\begin{array}{l}\text { Trois } \\
\text { dimensions } \\
\text { de notre } \\
\text { expérience } \\
\text { du monde: }\end{array}$ & $\begin{array}{c}\text { Trois } \\
\text { dimensions } \\
\text { du sens : }\end{array}$ & $\begin{array}{c}\text { Pour chaque } \\
\text { dimension } \\
\text { du sens, } \\
\text { un double } \\
\text { horizon cons- } \\
\text { tituté par une } \\
\text { distinction: }\end{array}$ & $\begin{array}{c}\text { Ce que } \\
\text { le système } \\
\text { observe, } \\
\text { auquel des } \\
2 \text { horizons } \\
\text { l'attribue-t-il? }\end{array}$ & $\begin{array}{l}\text { Les opéra- } \\
\text { tions signi- } \\
\text { fiantes du } \\
\text { système sont } \\
\text { déclenchées } \\
\text { par des } \\
\text { problèmes : }\end{array}$ & $\begin{array}{c}\text { Il y a } \\
\text { généralisation } \\
\text { symbolique }^{7} \\
\text { du sens } \\
\text { sous forme } \\
\text { d'attentes }\end{array}$ \\
\hline \multirow[t]{2}{*}{$\begin{array}{l}\text { les objets } \\
\text { matériels }\end{array}$} & \multirow{2}{*}{\begin{tabular}{|l} 
Dimension \\
de l'état \\
de choses
\end{tabular}} & interne & \multirow{2}{*}{$\begin{array}{l}\text { à ce qui } \\
\text { se passe } \\
\text { à l'intérieur } \\
\text { de lui ou } \\
\text { à l'extérieur } \\
\text { de lui? }\end{array}$} & \multirow{2}{*}{$\begin{array}{l}\text { de disjonction } \\
\text { primaire } \\
\text { (A différent } \\
\text { de B) }\end{array}$} & \multirow{2}{*}{$\begin{array}{l}\text { généralisations } \\
\text { selon l'état } \\
\text { de choses }\end{array}$} \\
\hline & & externe & & & \\
\hline \multirow[t]{2}{*}{ le temps } & \multirow{2}{*}{$\begin{array}{l}\text { Dimension } \\
\text { temporelle }\end{array}$} & passé & \multirow{2}{*}{$\begin{array}{l}\text { à un facteur } \\
\text { constant ou } \\
\text { variable? }\end{array}$} & \multirow{2}{*}{$\begin{array}{l}\text { liés à } \\
\text { l'irréversibilité } \\
\text { du temps }\end{array}$} & \multirow{2}{*}{$\begin{array}{l}\text { généralisations } \\
\text { dans le temps }\end{array}$} \\
\hline & & futur & & & \\
\hline \multirow[t]{2}{*}{ le social } & \multirow[t]{2}{*}{$\begin{array}{l}\text { Dimension } \\
\text { sociale }\end{array}$} & ego ou & \multirow{2}{*}{$\begin{array}{l}\text { à lui-même } \\
\text { ou à un autre } \\
\text { que lui? }\end{array}$} & \multirow{2}{*}{$\begin{array}{l}\text { liés à des } \\
\text { tiraillements } \\
\text { internes ou } \\
\text { des désaccords } \\
\text { avec autrui }\end{array}$} & \multirow{2}{*}{$\begin{array}{l}\text { généralisations } \\
\text { d'ordre social } \\
\text { (ex. : langage, } \\
\text { moralité) }\end{array}$} \\
\hline & & alter & & & \\
\hline
\end{tabular}


Tableau 3 - CONDENSATION DU SENS SELON TROIS DIMENSIONS

\begin{tabular}{|l||l|l|l|}
\hline $\begin{array}{l}\text { Trois } \\
\text { dimensions } \\
\text { du sens }\end{array}$ & $\begin{array}{l}\text { Les thèmes choisis } \\
\text { (ou sélections) } \\
\text { thématiques }\end{array}$ & $\begin{array}{l}\text { Condensations } \\
\text { structurelles de } \\
\text { thèmes dans chacune } \\
\text { des dimensions }\end{array}$ & $\begin{array}{l}\text { Des réservoirs } \\
\text { de thèmes }\end{array}$ \\
\hline $\begin{array}{l}\text { l'état } \\
\text { de choses }\end{array}$ & $\begin{array}{l}\text { Sur quoi porte la } \\
\text { contribution à la } \\
\text { communication? }\end{array}$ & $\begin{array}{l}\text { condensations struc- } \\
\text { turelles au niveau de } \\
\text { ceci plutôt que cela } \\
\text { (factual structures) }\end{array}$ & $\begin{array}{l}\text { Pour un choix plus } \\
\text { rapide et plus facile }\end{array}$ \\
\hline le temps & $\begin{array}{l}\text { Les thèmes peuvent être } \\
\text { anciens ou nouveaux. }\end{array}$ & $\begin{array}{l}\text { condensations } \\
\text { structurelles } \\
\text { dans le temps } \\
\text { (temporal structures) }\end{array}$ & $\begin{array}{l}\text { Les thèmes peuvent } \\
\text { atteindre un point } \\
\text { de saturation à par- } \\
\text { tir duquel aucune } \\
\text { nouvelle contribu- } \\
\text { tion n'est attendue. }\end{array}$ \\
\hline le social & $\begin{array}{l}\text { La sélection s'actualise dans } \\
\text { cette dimension lorsque } \\
\text { la communication en vient } \\
\text { à lier entre eux les partici- } \\
\text { pants. Cela veut alors dire } \\
\text { qu'ils ont exprimé quel- } \\
\text { que chose au sujet d'eux- } \\
\text { mêmes, de leurs opinions, } \\
\text { attitudes, expériences, } \\
\text { souhaits ou intérêts. }\end{array}$ & $\begin{array}{l}\text { condensations } \\
\text { structurelles servant } \\
\text { à la communication } \\
\text { (social structures) }\end{array}$ & $\begin{array}{l}\text { la sémantique } \\
\text { en tant que } \\
\text { réservoir de } \\
\text { thèmes pour la } \\
\text { communication }\end{array}$ \\
\hline
\end{tabular}

* C'est à la présentation d'une telle sémantique qu'est consacré l'article de Klaus Dammann sur guerre, génocide et terrorisme, dans ce numéro.

Luhmann précise qu'il s'abstient de puiser dans le structuralisme et dans le fonctionnalisme structurel pour élaborer ce qu'il entend par structure d'un système autoréférentiel et autopoïétique (1995, p. 278-280). Par ailleurs, la notion de structure en herméneutique mérite d'être rappelée ici car son introduction par Dilthey a inauguré une nouvelle forme d'explication des faits, en dehors du principe de causalité. Dans un cours donné en 1988, Gadamer est éloquent à ce sujet : «Dilthey fait intervenir [...] le concept d'un "système d'effets" qui vise non pas la distinction entre cause et effet mais la mise en évidence de ce qui lie les uns aux autres des effets qui sont tous en relation mutuelle. Ce qui est le cas non seulement de l'organisme vivant mais également de l'œuvre d'art. L'exemple favori de Dilthey est la structure d'une mélodie» (2001, p. 29).

Luhmann ne parle pas d'un système d'effets, mais il définit la notion de système sans faire référence à autre chose que des opérations interconnectées: "quand des opérations se connectent les unes aux autres, un système advient » (Luhmann, cité dans Clam, 1997, p 254).

Sur la notion de structure chez Dilthey, Gadamer écrit :

ce qui est à mon sens fondamental chez Dilthey, c'est-à-dire le concept de structure, naturellement pris dans l'intégralité de son sens et non pas au sens particulier qu'il a pour le structuralisme aujourd'hui. L'introduction, réalisée par Dilthey, du concept de structure dans la discussion philosophique est un fait remarquable. [...] En un temps où la théorie de la connaissance occupait une position dominante, Dilthey a osé se dresser contre la tendance à voir dans la logique de l'induction et dans le principe de causalité l'unique forme d'explication des faits.

Le terme de «structure» signifie dans ce cadre qu'il y a une autre manière de comprendre la vérité que celle de la recherche causale. Il indique la solidarité des parties, parmi lesquelles aucune n'est conçue comme ayant préséance sur les autres. [.... Le terme de structure veut dire qu'il n'y a pas d'abord une cause et ensuite un effet mais qu'il s'agit au contraire d'un jeu commun d'effets. (2001, p. 28-29).

Un système autopoiétique est, on l'a $v u$, à la fois la cause et l'effet de ce qu'il est. Retenons l'expression de Gadamer et essayons de voir comment pourrait se manifester un tel jeu commun d'effets dans un système récursif. Jean Clam présente la structures des systèmes autopoiétiques chez Luhmann comme une "prise d'appui du système sur le flux opératif qui le constitue » et un « rejet structurel et permanent» du système sur lui-même. Les opérations du système étant récursives, le système " prend à chaque fois son départ dans les opérations qui viennent de tomber dans l'inactualité et les structures qui fondent les expectations en cours [...] toute connexion est sélective, c'est-à-dire qu'elle prend en compte les opérations qui précèdent et celles qui suivent. Les condensations structurelles aiguillent le système et orientent sa récursivité » (1997, p. 258-259).

Nous avons déjà présenté le processus de généralisation du sens dans des attentes. Lors des opérations de communication, il y a généralisation du sens dans ce que Luhmann appelle des thèmes, ou " contenu attendu » de la communication (1995, p. 157). Grâce à ces généralisations, le système social en cause accroît ses chances que sa communication réussisse à se connecter à d'autres communications et que le système puisse poursuivre son autopoiièse. Un thème est une généralisation du sens : il indique sur quoi la sélection va porter mais laisse ouvert tout un champ de possibilités quant à savoir quelles contributions pourront être sélectionnées, quand, dans quel ordre et par qui. Le thème donne suffisamment d'indications sur le sens qui se constitue par la communication pour permettre aux systèmes en présence de choisir rapidement leur contribution à la communication, d'évaluer si la contribution de l'autre est adéquate, bref de s'orienter l'un par rapport à l'autre selon qu'ils souhaitent poursuivre ou interrompre la communication.

Dans les opérations de communication, les thèmes servent de structure ${ }^{9}$; ils peuvent donner lieu à des condensations structurelles dans chacune des trois dimensions du sens (tableau 3).

Une fois constituées, les structures deviennent ce sur quoi s'orientent les opérations des systèmes autopoiétiques. Mais il ne faut pas oublier qu'il ne s'agit pas de structures-objets, mais d'un jeu d'effets, en situation de complexité.

La théorie de Niklas Luhmann sur la constitution du sens est fascinante et il est certain qu'une présentation aussi rapide ne saurait lui rendre justice. Les trois dimensions du sens ont été trop brièvement esquissées ici sous forme de tableaux et mériteraient un développement plus élaboré. L'exercice n'aura cependant pas été vain s'il permet d'attirer l'attention sur la portée de l'autopoïèse axée sur le sens, c'est-à-dire de l'autoproduction de soi dans et par la production du sens. La constitution du sens grâce à nos opérations conscientes et à nos communications n'est pas un banal processus, parmi tant d'autres. Ce qui est en jeu, c'est qui nous sommes, qui nous devenons en communiquant les uns avec les autres. S'il y a inscription corporelle du sens, nous en sommes le lieu vivant. 


\section{Bibliographie}

CLAM, Jean (1997). Droit et société chez Niklas Luhmann, Paris, PUF

DEPRAZ, Natalie (1999). Husserl, Paris, Armand Colin.

DESANTI, Jean Toussaint (1976 et 1994), Introduction à la phénoménologie, Paris, Gallimard.

GADAMER, Hans-Georg (2001). Au commencement de la philosophie, Paris, Seuil.

HOUSSET, Emmanuel (2000). Husserl et l'énigme du monde, Paris, Seuil.

HUSSERL, Edmund (1950). Idées directrices pour une phénoménologie, trad. P. Ricoeur, Paris, Gallimard.

HUSSERL, Edmund (1994). Méditations cartésiennes, trad. M. de Launay, Paris, PUF.

LUHMANN, Niklas (2000). The Reality of the Mass Media, Stanford, Stanford University Press.

LUHMANN, Niklas (1995). Social Systems, Stanford, Stanford University Press.

LUHMANN, Niklas, (1994). "Entretien entre N. Luhmann et D. Sciulli», dans Theory, Culture and Society, Londres, vol. 11, $\mathrm{n}^{\circ} 2$, p. 37.

LUHMANN, Niklas (1990a). Essays on Self-Reference, New York, Columbia University Press.

LUHMANN, Niklas (1990b). "Développements récents en théorie des systèmes ", dans G. Duprat, Connaissance du politique, Paris, PUF.

RICHIR, Marc (2001). "La phénoménologie de Husserl dans la philosophie de MerleauPonty. Questions phénoménologiques », dans J.-F. Mattéi, Philosopher en français, Paris, PUF.

SALANSKIS, Jean-Michel (1997). «Herméneutique et philosophie du sens », dans J.-M. Salanskis, F. Rastier et R. Scheps, Herméneutique: textes, sciences, Cerisy, Paris, PUF.

\section{Notes}

1. Voir à ce sujet le texte de l'entretien entre N. Luhmann et D. Sciulli, dans Theory, Culture and Society, Londres, vol. 11, $\mathrm{n}^{\circ} 2$, 1994, p. 37, où Luhmann précise qu'à cause de ses circu-larités (its in-built circularities) le concept de sens peut contribuer à une démarche d'intégration entre la théorie des systèmes et les descriptions herméneutiques (phénoménologiques) et qu'il s'impose dès lors comme un concept de base pour la sociologie. Luhmann donne en référence son chapitre 2 de Essays on Self-Reference, p. 21 à 79, intitulé: "Meaning as Sociology's Basic Concept », écrit en allemand en 1969.

2. La discussion autour de la notion d'autopoièse a d'abord été proposée par les neurobiologistes Humberto Maturana et Francisco Varela, dans les années 1960. L'organisation autopoïétique d'un système serait selon eux le critère qui permet d'établir que l'on est en présence d'un système vivant. Luhmann a repris la notion d'autopoièse pour l'élargir à la «production » non seulement de la vie mais du sens.

3. «Rhumbs», dans EEuvres, Paris, la Pléiade, vol. 2, 1960, p. 611 .

4. Un bref rappel: «Transcendantal. Le mot est ancien. Mais son sens moderne a été élaboré par Kant. Celui-ci appelle "transcendantales" les conditions nécessaires et universelles qui fondent a priori la possibilité de l'expérience. Ainsi pour lui l'espace est une condition transcendantale de la perception des objets extérieurs.

Le sens kantien est passé chez Husserl. La phénoménologie est une "philosophie transcendantale": elle cherche les structures universelles et $a$ priori qui permettent la détermination du sens de toute expérience possible pour une conscience. Bien entendu, ces "conditions transcendantales" sont, pour Husserl, bien différentes de ce qu'elles étaient chez Kant », Desanti, 1994, Glossaire, p. 170.
5. Un bref rappel : "Constitution. Constituer consiste à donner sens à ce qui se présente. Tout objet est un sens constitué par des noèses. La constitution n'est donc pas ici l'acte de produire un objet dans le monde, mais l'acte par lequel un sens d'objet se forme dans le cours de l'expérience. » Housset, 2000, Glossaire, p. 259. Par ailleurs, Husserl parle de «donation » pour décrire le «processus par lequel un objet ou un vécu advient à ma conscience, qui lui donne alors sens. Alors que «constitution» souligne l'activité d'appropriation de l'objet par le sujet, donation conjugue passivité et activité : l'objet $m$ 'est donné en lui-même», Depraz, 1999, Glossaire, p. 90.

6. Un processus c'est, pour Luhmann, une liaison établie dans le temps entre une pluralité d'événements qui sont eux-mêmes des sélections, par le jeu de conditionnements réciproques (1995, p. 154).

7. L'adjectif symbolique mériterait des explications dans lesquelles le cadre limité de cet article ne permet pas de s'engager. Je me contente donc de citer en anglais la présentation qu'en fait Luhmann: "The concept symbol/ symbolic in that indicates the medium in which units are formed; the concept of generalization the units' function - to handle multiplicity operatively. In very rough outlines, it is a matter of a plurality being related to a unity and symbolized by it. Thereby a difference between operative (or processual) and symbolic levels emerges that makes self-referential operations actually possible» (1995, p. 93).

8. C'est par un exemple amusant, choisi au niveau des faits concrets, que Luhmann précise cette déclaration: vous entendez le bruit que font dans votre ruelle les vidangeurs et leur camion, vous comprenez que votre poubelle a été vidée et vous allez la chercher et, même si elle n'a pas de nom, vous la reconnaissez parmi les autres poubelles qui se trouvent dans la ruelle (1995, p. 94).

9. On comprend dès lors que Klaus Dammann, dans l'article publié dans ce numéro, puisse nous proposer d'observer le terrorisme en tant que structure sociale et comme un cas spécial de la communication militaire. 